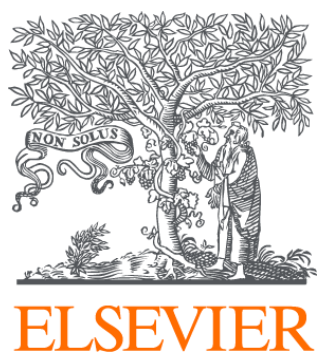

Since January 2020 Elsevier has created a COVID-19 resource centre with free information in English and Mandarin on the novel coronavirus COVID-

19. The COVID-19 resource centre is hosted on Elsevier Connect, the company's public news and information website.

Elsevier hereby grants permission to make all its COVID-19-related research that is available on the COVID-19 resource centre - including this research content - immediately available in PubMed Central and other publicly funded repositories, such as the WHO COVID database with rights for unrestricted research re-use and analyses in any form or by any means with acknowledgement of the original source. These permissions are granted for free by Elsevier for as long as the COVID-19 resource centre remains active. 


\title{
Explosive nosocomial outbreak of SARS-CoV-2 in a rehabilitation clinic: the limits of genomics for outbreak reconstruction
}

\author{
M. Abbas ${ }^{a, b, *}$, T. Robalo Nunes ${ }^{a, c}$, A. Cori ${ }^{\text {b, d }}$, S. Cordey ${ }^{e, f}$, F. Laubscher ${ }^{e}$, \\ S. Baggio ${ }^{g, h}$, T. Jombart ${ }^{d, i}$, A. Iten ${ }^{a}$, L. Vieux ${ }^{j}$, D. Teixeira ${ }^{a}$, M. Perez ${ }^{a}$, \\ D. Pittet $^{a, f}$, E. Frangos ${ }^{k}$, C.E. Graf ${ }^{\prime}$, W. Wingg ${ }^{a, m, \dagger}$, S. Harbarth ${ }^{a, f, \dagger}$ \\ a Infection Control Programme, Geneva University Hospitals, Geneva, Switzerland \\ ${ }^{\mathrm{b}} \mathrm{MRC}$ Centre for Global Infectious Disease Analysis, Imperial College London, London, UK \\ ' Serviço de Infecciologia, Hospital Garcia de Orta, EPE, Almada, Portugal \\ ${ }^{\mathrm{d}}$ The Abdul Latif Jameel Institute for Disease and Emergency Analytics, School of Public Health, Imperial College London, \\ London, UK \\ e Laboratory of Virology, Department of Diagnostics, Geneva University Hospitals, Geneva, Switzerland \\ ${ }^{f}$ Faculty of Medicine, University of Geneva, Geneva, Switzerland \\ ${ }^{\mathrm{g}}$ Division of Prison Health, Geneva University Hospitals, Geneva, Switzerland \\ ${ }^{\mathrm{h}}$ Office of Correction, Department of Justice and Home Affairs of the Canton of Zurich, Zurich, Switzerland \\ ${ }^{i}$ Centre for Mathematical Modelling of Infectious Diseases, Department of Infectious Disease Epidemiology, London School of \\ Hygiene and Tropical Medicine, London, UK \\ j Occupational Health Service, Geneva University Hospitals, Geneva, Switzerland \\ ${ }^{\mathrm{k}}$ Clinique de Joli-Mont, Department of Rehabilitation and Geriatrics, Geneva University Hospitals, Geneva, Switzerland \\ 'Department of Rehabilitation and Geriatrics, Geneva University Hospitals, Geneva, Switzerland \\ $\mathrm{m}$ Infection Control Programme, Zurich University Hospital, Zurich, Switzerland
}

\section{A R T I C L E I N F O}

\section{Article history:}

Received 25 June 2021

Accepted 27 July 2021

Available online 27 August 2021

\section{Keywords:}

COVID-19

SARS-CoV-2

Infection prevention and

control

Healthcare-associated infection

Nosocomial outbreaks

Long-term care facilities
$S \cup M M A R$

Background: Nosocomial outbreaks of severe acute respiratory syndrome coronavirus-2 (SARS-CoV-2) are frequent despite implementation of conventional infection control measures. An outbreak investigation was undertaken using advanced genomic and statistical techniques to reconstruct likely transmission chains and assess the role of healthcare workers (HCWs) in SARS-CoV-2 transmission.

Methods: A nosocomial SARS-CoV-2 outbreak in a university-affiliated rehabilitation clinic was investigated, involving patients and HCWs, with high coverage of pathogen wholegenome sequences (WGS). The time-varying reproduction number from epidemiological data $\left(R_{t}\right)$ was estimated, and maximum likelihood phylogeny was used to assess genetic diversity of the pathogen. Genomic and epidemiological data were combined into a Bayesian framework to model the directionality of transmission, and a case-control study was performed to investigate risk factors for nosocomial SARS-CoV-2 acquisition in patients.

* Corresponding author. Address: Infection Control Programme, Geneva University Hospitals and Faculty of Medicine, Rue Gabrielle-Perret-Gentil

4, 1211 Geneva, Switzerland. Tel.: +41223728828.

E-mail address: mohamed.abbas@hcuge.ch (M. Abbas).

$\dagger$ These authors contributed equally. 
Findings: The outbreak lasted from $14^{\text {th }}$ March to $12^{\text {th }}$ April 2020, and involved 37 patients ( 31 with WGS) and 39 employees ( 31 with WGS), 37 of whom were HCWs. Peak $R_{t}$ was estimated to be between 2.2 and 3.6. The phylogenetic tree showed very limited genetic diversity, with 60 of $62(96.7 \%)$ isolates forming one large cluster of identical genomes. Despite the resulting uncertainty in reconstructed transmission events, the analyses suggest that HCWs (one of whom was the index case) played an essential role in crosstransmission, with a significantly greater fraction of infections $(P<2.2 \mathrm{e}-16)$ attributable to HCWs $(70.7 \%)$ than expected given the number of HCW cases $(46.7 \%)$. The excess of transmission from HCWs was higher when considering infection of patients $[79.0 \%$; $95 \%$ confidence interval $(\mathrm{Cl})$ 78.5-79.5\%] and frail patients (Clinical Frailty Scale score $>5$; 82.3\%; 95\% Cl 81.8-83.4\%). Furthermore, frail patients were found to be at greater risk for nosocomial COVID-19 than other patients (adjusted odds ratio 6.94, 95\% Cl 2.13-22.57). Interpretation: This outbreak report highlights the essential role of HCWs in SARS-CoV-2 transmission dynamics in healthcare settings. Limited genetic diversity in pathogen genomes hampered the reconstruction of individual transmission events, resulting in substantial uncertainty in who infected whom. However, this study shows that despite such uncertainty, significant transmission patterns can be observed.

(c) 2021 The Authors. Published by Elsevier Ltd on behalf of The Healthcare Infection Society. This is an open access article under the CC BY-NC-ND license (http://creativecommons.org/licenses/by-nc-nd/4.0/).

\section{Introduction}

Nosocomial outbreaks of severe acute respiratory syndrome coronavirus-2 (SARS-CoV-2) are frequent, and place an even greater burden on healthcare systems coping with the coronavirus disease 2019 (COVID-19) pandemic [1]. Long-term care facilities (LTCFs) and nursing homes have borne the brunt of these nosocomial outbreaks, with a devastating impact on morbidity and mortality [2-4].

These nosocomial outbreaks represent a challenge for infection prevention and control (IPC) professionals as they have been widespread despite implementation of traditional preventive measures. Among the hypotheses for the origins of these outbreaks are the challenges of asymptomatic and pre-symptomatic transmission of SARS-CoV-2 [3,5], and the role of healthcare workers (HCWs) as vectors and victims of these outbreaks, as they are at the interface between healthcare settings and the community, and are not subject to strict lockdown measures [6]. Although several reports have used whole-genome sequencing (WGS) and epidemiological data to describe outbreaks, very few have also included data and sequences from HCWs [7-12].

The aim of this study was to reconstruct a nosocomial outbreak in a rehabilitation clinic during the first wave of the COVID-19 pandemic, combining epidemiological and genetic data, in order to better understand transmission dynamics. Specific objectives were to establish viral transmission pathways, reconstruct a transmission tree, identify the major modes of directionality of transmission, assess the impact of infection prevention and control (IPC) measures on controlling the outbreak, and evaluate risk factors for nosocomial acquisition of SARS-CoV-2.

\section{Methods}

A retrospective description and analysis of a SARS-CoV-2 outbreak involving patients and HCWs in one of the rehabilitation clinics of Geneva University Hospitals (HUG) was performed. The report was written in accordance with the ORION guidelines [13].

\section{Setting}

HUG is a tertiary care hospital consortium in Western Switzerland. As part of HUG, there is a five-ward LTCF with 88 beds catering for patients requiring palliative or rehabilitation care, or for patients awaiting placement in a nursing home. The rehabilitation clinic also has a restaurant, which serves meals to patients, HCWs and external visitors. This clinic is monitored directly by the IPC Programme staff, with weekly in-person visits by members of staff and daily discussion of IPC measures by telephone.

\section{Definition of cases}

Nosocomial COVID-19 was defined as patients with a positive reverse transcription polymerase chain reaction (RT-PCR) for SARS-CoV-2 with onset of symptoms compatible with SARS-CoV2 infection $\geq 5$ days after admission to the clinic, in accordance with Swissnoso guidelines [14]. HCWs were included in the outbreak study if they were employed in the rehabilitation clinic during the study period, and presented a positive RT-PCR for SARS-CoV-2.

\section{Data sources}

Data collected as part of prospective surveillance of all patients hospitalized with COVID-19 mandated by the Swiss Federal Office of Public Health (FOPH) were used in this study [15]. During the first wave of COVID-19, the HUG Department of Occupational Health collected data prospectively from HCWs with a positive RT-PCR for SARS-CoV-2, including date of symptom onset and potential contacts. In addition, HUG offered free RT-PCR testing for HCWs in a designated testing site, and collected data on all individuals who presented for SARS-CoV-2 testing as part of a prospective cohort study. Data were extracted from the HUG Department of Human Resources on HCW shifts. Patient trajectories were extracted from the electronic health records. 
For the case-control study, data were collected retrospectively from the electronic health records for the control group, using the same RedCAP ${ }^{T M}$ eCRF as for the FOPH cohort study. Additional data collection instruments were created in order to capture relevant risk factors based on an informal literature search for both cases and controls. Among the variables collected were the Clinical Frailty Scale (CFS) score [16], hypoalbuminaemia (and hypoprealbuminaemia) as a proxy for malnutrition [17], current smoking, age, body mass index, comorbidities, the Charlson Comorbidity Index [18], and the Cumulative Illness Rating Scale - Geriatric (CIRS-G) [19]. The CIRS-G was dichotomized as a binary variable with a cut-off at 15 points [20].

\section{Descriptive epidemiology}

An epidemic curve was produced using dates of symptom onset; where these were unavailable (e.g. lack of symptoms), they were imputed with the median difference between date of symptom onset and date of nasopharyngeal swab.

\section{Microbiological methods}

All COVID-19 cases in the outbreak were confirmed by RTPCR on nasopharyngeal swabs. Three diagnostic methods were used for routine screening: the Cobas 6800 SARS CoV2 RTPCR (Roche, Basel, Switzerland), the BD SARS-CoV2 reagent kit for BD Max system (Becton, Dickinson and Co, Franklin Lakes, NJ, USA), and an in-house method based on the Charité assay [21]. SARS-CoV-2 WGS was performed using either an unbiased high-throughput sequencing method or an amplicon-based sequencing method in order to produce RNA sequences. Full details of the microbiological methods, including the WGS and the sequence assembly, can be found in the online supplementary material.

\section{Phylogenetic analysis}

Sequence alignment was performed using MUSCLE v3.8.31. The evolutionary analyses were conducted in MEGA X [22] using the maximum likelihood method and Tamura 3-parameter model [23]. All SARS-CoV-2 complete genomes sequenced by the Laboratory of Virology (HUG) in the context of epidemiological surveillance and submitted to Global Initiative on Sharing Avian Influenza Database (GISAID) from respiratory samples from patients with COVID-19 were integrated to the phylogenetic analysis in addition to the case samples of this outbreak analysis. The samples represent tests from various medical centres in Geneva, Switzerland during the same period.

\section{Statistical analysis}

Descriptive statistics were performed with medians and interquartile ranges (IQR), and counts and proportions, as appropriate. A case-control study was performed to identify risk factors for nosocomial COVID-19 among patients. The control population was defined as patients who were hospitalized for $\geq 5$ days (to avoid immortal bias) in the rehabilitation clinic during the outbreak period, and who had neither a positive RT-PCR for SARS-CoV-2 nor a clinical diagnosis of COVID-19 (presumed or confirmed). Unmatched controls were selected at random from this population at a ratio of 2:1. Associations between patient characteristics and nosocomial COVID-19 were assessed using the Chi-squared test for categorical variables, and the Kruskall-Wallis test for continuous variables. Univariable logistic regression was performed to produce crude odds ratios (OR) and 95\% confidence intervals $(95 \% \mathrm{Cl})$. A multi-variable logistic regression model was built using a forward fitting method, and the most parsimonious model was selected as guided by the Akaike Information Criterion and likelihood ratio tests [24]. Goodness-of-fit was evaluated using the Hosmer-Lemeshow test.

The time-varying reproduction number $\left(R_{t}\right)$ was estimated from the incidence of symptoms, using the incidence and EpiEstim packages for the R statistical software [25]. $R_{t}$ estimation was performed under a 'short' serial interval (time between onset of symptoms in an infector/infectee pair) assumption, consistent with early isolation of cases in the hospital context. A 'long' serial interval assumption was considered in sensitivity analyses (see online supplementary material).

\section{Reconstruction of transmission trees}

Epidemiological and genetic data were combined to reconstruct who infected whom using the R package outbreaker2, which has been used successfully to reconstruct 2003 SARSCoV-1 transmission trees [26,27]. The model uses a Bayesian framework, which combines information on the generation time (time between infections in an infector/infectee pair) with a model of sequence evolution to probabilistically reconstruct the transmission tree (see online supplementary material for details on the analyses performed).

The method reflects the uncertainty in individual transmission events by producing a large number of trees (posterior trees) compatible with the data. To assess the role of HCWs in transmission, $f_{H C W}$, the proportion of infections attributed to HCWs for each of the 999 posterior trees retained, was derived. We tested if the average value of $f_{H C W}$ was greater than expected given the proportion of HCWs amongst cases using a Wilcoxon-Mann-Whitney test. This analysis was done for all cases, and also stratified by type of case infected: other HCWs, patients, and frail patients (CFS score $>5$ ). A sensitivity analysis was conducted by replicating these analyses on runs of outbreaker 2 , including one where contact data were not used in the transmission tree reconstruction.

Statistical analyses were performed using $\mathrm{R}$ software version 4.0.3 (https://www.R-project.org/) and Stata Version 14 (StataCorp, College Station, TX, USA).

\section{Ethical considerations}

Outbreak investigations are within the purview of the IPC programme. For additional investigations, the local ethics committee approved this study (Ref. No. 2020-01330).

\section{Results}

The index case of the outbreak was a HCW who was detected on $14^{\text {th }}$ March 2020; the first patient was detected 5 days later on the same ward, and the last case was detected on $17^{\text {th }}$ April 2020. Therefore, the outbreak period was defined as lasting from $1^{\text {st }}$ March 2020 to $19^{\text {th }}$ April 2020. In total, 37 
patients and 39 hospital employees (including two administrative staff) were involved in the outbreak. The institutionlevel attack rate for patients was $21.2 \%$. The epidemic curve is shown in Figure 1A. All five wards of the clinic were involved in the outbreak. Characteristics of patients and employees are summarized in Table I and Table II, respectively.

Several infection control measures were implemented sequentially (Figure $1 \mathrm{C}$ ). Staff with a positive SARS-CoV-2 RTPCR were immediately placed on mandatory sick leave for a minimal duration of 10 days from the onset of symptoms. Two ward-level point-prevalence screening surveys of all negative patients (including asymptomatic) were performed in affected wards, and all staff were strongly encouraged to undergo testing. Universal screening on admission was performed from $2^{\text {nd }}$ April 2020. Throughout the outbreak period, RT-PCR results were obtained with an average turnaround time of approximately $8 \mathrm{~h}$.

\section{Estimated time-varying reproduction number}

It was estimated that $R_{t}$ declined rapidly from 2.17 [95\% credible interval $(\mathrm{Crl}) 1.43-3.07]$ by $21^{\text {st }}$ March 2020 , with mean $R_{t}$ reaching $<1$ on $28^{\text {th }}$ March 2020 (Figure 1B). Trends were similar when assuming a longer serial interval, albeit with higher mean $R_{t}$ estimates, at 3.60 (95\% Crl 2.37-5.08) initially and $<1$ by $28^{\text {th }}$ March 2020 (Supplementary Figure 1, see online supplementary material). Confining patients to their room decreased $R_{t}$, but not below 1 ; although this was achieved by

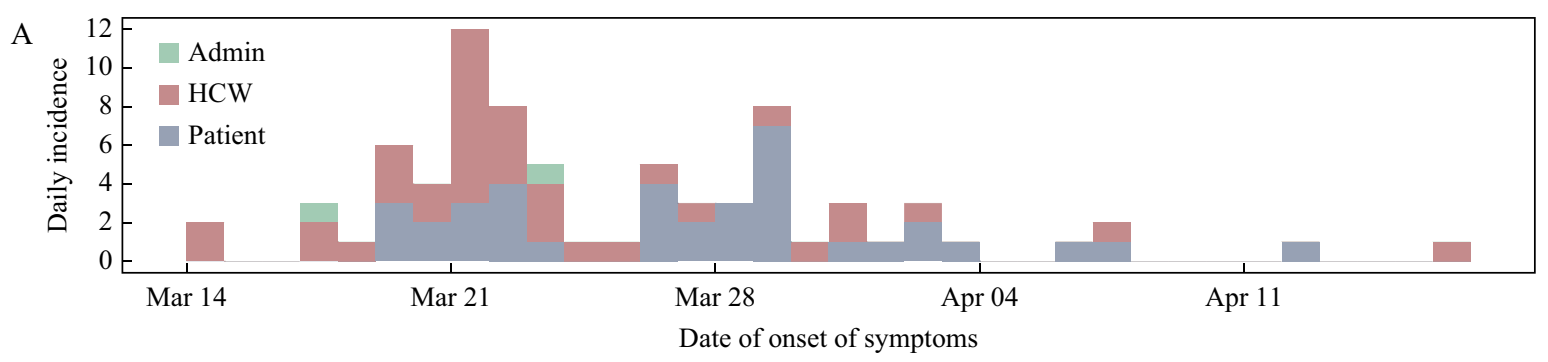

B
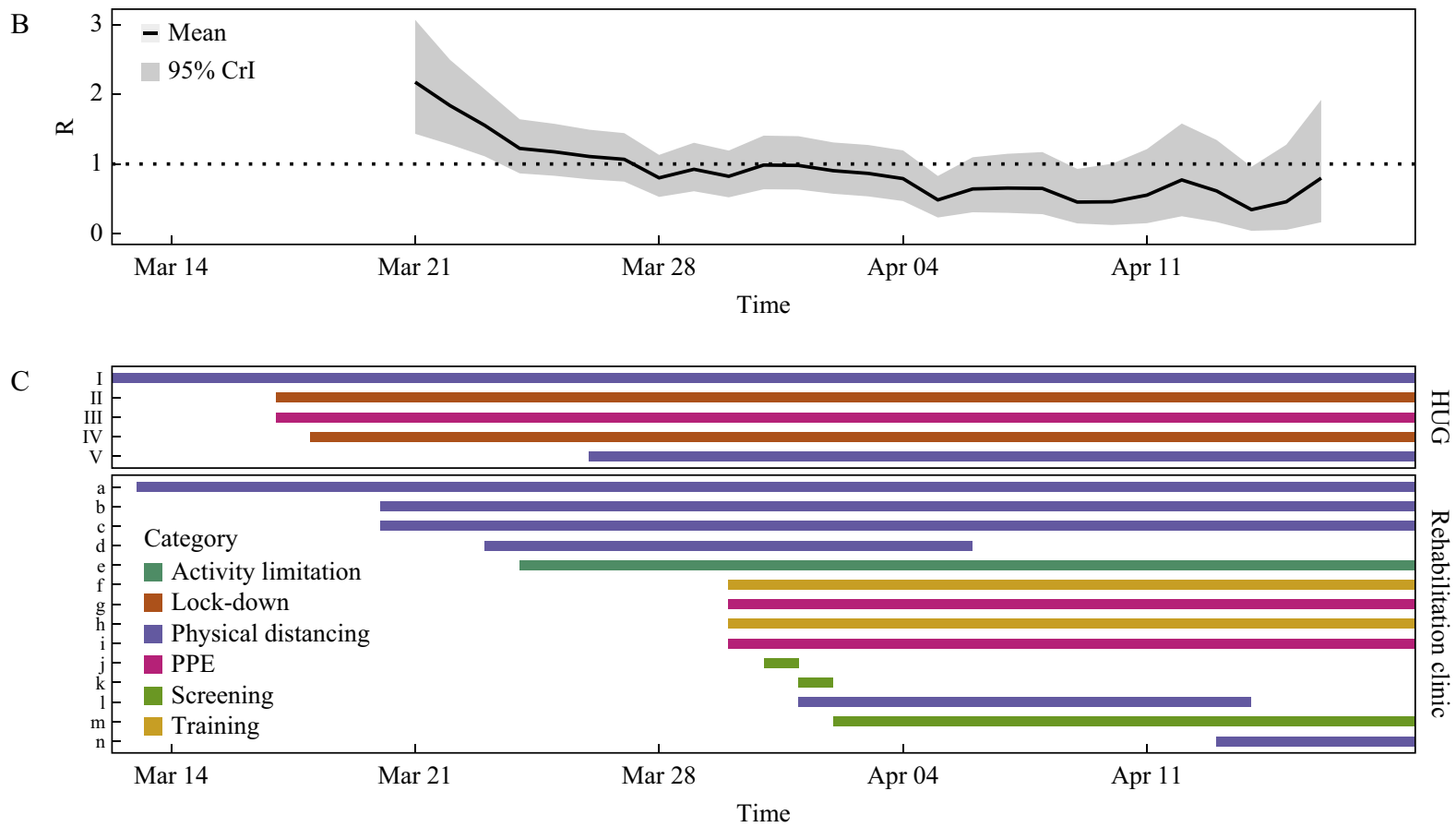

Figure 1. (A) Epidemic curve of the nosocomial outbreak of coronavirus disease 2019 (COVID-19) in a rehabilitation clinic involving healthcare workers (HCWs) and patients. (B) Estimated time-varying reproduction number $\left(R_{t}\right)$ across the duration of the outbreak. (C) Timeline of infection prevention and control interventions implemented hospital-wide (Geneva University Hospitals), and specifically in the rehabilitation clinic. I, group activities with patients suspended; II, cafeterias only open to employees; III, universal masking (HCWs); IV, visitors banned; V, physical distancing in cafeterias; a, meals in rooms for patients; b, room confinement for patients on second floor; $c$, single bed rooms only (second floor); $d$, ward closure on second floor; e, ward attribution to physical therapists; $f$, daily meetings with infection prevention and control (IPC) nurse; g, pre-emptive contact and droplet precautions in wards with at least one patient with COVID-19; $h$, reminder of IPC measures; $i$, universal masking outside room (patients); $j$, systematic severe acute respiratory syndrome coronavirus-2 (SARS-CoV-2) screening of all patients on second floor; k, systematic SARS-CoV-2 screening of all patients on first floor; l, ward closure on first floor; $m$, systematic SARS-CoV-2 screening of all admissions; $n$, ward closure ground floor. PPE, personal protective equipment; $\mathrm{Crl}$, credible interval. 
Table I

Characteristics of patients

\begin{tabular}{|c|c|c|c|}
\hline Characteristic & $\begin{array}{c}\text { Nosocomial COVID-19 } \\
\text { patients }(N=37)\end{array}$ & $\begin{array}{c}\text { Uninfected } \\
\text { controls }(N=74)\end{array}$ & $P$-value \\
\hline Age, years & 87 (77-89) & $80(74-86)$ & 0.03 \\
\hline Obesity & $4(10.8)$ & $15(20.3)$ & 0.21 \\
\hline Active smoking & $1(2.7)$ & $12(16.2)$ & 0.04 \\
\hline Clinical Frailty Scale score $>5$ & $33(89.2)$ & $40(54.1)$ & $<0.001$ \\
\hline Cumulative Illness Rating Scale - Geriatric & $14(12-18)$ & $15(11-19)$ & 0.98 \\
\hline Cumulative Illness Rating Scale - Geriatric $\geq 15$ & $18(48.7)$ & $38(51.4)$ & 0.79 \\
\hline Charlson Comorbidity Index & $7(4-9)$ & $6(4-8)$ & 0.30 \\
\hline Charlson Comorbidity Index $>7$ & $17(46.0)$ & $19(25.7)$ & 0.03 \\
\hline Chronic respiratory disease & $6(16.2)$ & $25(33.8)$ & 0.04 \\
\hline Diabetes mellitus & $6(16.2)$ & $21(28.4)$ & 0.16 \\
\hline Hypertension & $25(67.6)$ & $52(70.3)$ & 0.77 \\
\hline Chronic cardiovascular disease & $17(46.0)$ & $36(48.7)$ & 0.79 \\
\hline Chronic renal disease & $7(18.9)$ & $14(18.9)$ & 1.00 \\
\hline Chronic liver disease & $1(2.7)$ & $5(6.8)$ & 0.37 \\
\hline Chronic neurological impairment & $12(32.4)$ & $16(21.6)$ & 0.22 \\
\hline Haematological disease with immunosuppression & 0 & $6(8.1)$ & 0.08 \\
\hline Cancer & $8(21.6)$ & $16(21.6)$ & 1.00 \\
\hline Rheumatological disease & 0 & $5(5.4)$ & 0.15 \\
\hline Dementia & $8(21.6)$ & $25(33.8)$ & 0.19 \\
\hline
\end{tabular}

HIV, human immunodeficiency virus; ACE, angiotensin-converting enzyme.

Results are expressed as median and interquartile range, or count and percentage.

${ }^{a}$ Missing for two patients.

b Missing for five patients.

c Missing for 23 patients.

closing the wards on the second floor, the upper limit of the $95 \%$ Crl was $>1$ (Supplementary Figure 2, see online supplementary material). Pre-emptive contact precautions and mandatory masking of patients outside of rooms decreased $R_{t}$ further, with the upper limit of the $95 \% \mathrm{Crl}<1$.

\section{Phylogenetic tree}

SARS-CoV-2 sequences were obtained for 62 cases (31 patients, 31 employees), and a phylogenetic tree was generated (Figure 2). Interestingly, although some tree branches should be interpreted with caution due to moderate bootstrap values reflecting the very high sequence homology between isolates, some branch-specific mutations (e.g. C5239T, C15324T or G29781T) were observed that support some branching order. Sixty of 62 sequences $(96.8 \%)$ formed a large single cluster with clear segregation from the community sequences. One large subcluster (bootstrap 60\%) was observed which corresponds to a specific ward in the clinic. Sequences from patients and HCWs were distributed similarly across all branches of the tree. The phylogenetic analysis suggests importation of two cases (3.2\%): H2030 (HCW) and H2013 (admin).

Table II

Characteristics of hospital employees

\begin{tabular}{ll}
\hline Characteristic & Hospital employees $(N=37)$ \\
\hline Age, years & $44(38-52)$ \\
Female & $33(84.6)$ \\
Profession & \\
$\quad$ Registered nurse & $15(38.5)$ \\
$\quad$ Nurse assistant & $13(33.3)$ \\
Doctor & $4(10.3)$ \\
Physical therapists & $3(7.7)$ \\
Administrative staff & $2(5.1)$ \\
Apprentice & $2(5.1)$ \\
Any symptoms & \\
Time from symptom to swab, days & $33(100 \%)$ \\
\hline
\end{tabular}

${ }^{a}$ Missing for four cases. 




Figure 2. Phylogenetic tree of severe acute respiratory syndrome coronavirus-2 (SARS-CoV-2) genome sequences. The tree includes 62 sequences related to the outbreak (patient and employee sequences are named C2xx and H20xx, respectively), alongside all the community cases in the canton of Geneva that were sequenced in March-April 2020 and submitted to GISAID [virus names and accession ID (i.e. EPI_ISL_) are indicated] in the context of an epidemiological surveillance. For each sequence, the date of the sample collection is mentioned (yyyy-mm-dd). 
outbreaker2 analysis

Figure 3 shows the main analysis (see Supplementary Figure 3 for Gelman-Rubin diagnostics, and Supplementary Figures 4-6 for sensitivity analyses). The probabilistic reconstruction of who infected whom demonstrates substantial uncertainty (Figure 3A), with only two and 12 ancestries (i.e. infectors) identified with $\geq 50 \%$ and $\geq 25 \%$ posterior probability, respectively. The model assumes that the first case in the outbreak is imported, and identified two additional imported cases. Both the ancestry plot (Figure $3 \mathrm{~A}$ ) and the highest posterior transmission tree (Figure $3 \mathrm{~B}$ ) suggest several super-spreading events, most likely originating from HCWs. Of the two infected administrative staff, one was detected as an imported case, and the other was estimated to have been infected by a HCW with a posterior probability of $81.6 \%$.

The proportion of infections attributed to HCWs (noted $\left.f_{\mathrm{HCW}}\right)$ was compared with random expectations assuming HCWs, and patients were equally likely to seed new infections. $f_{\mathrm{HCW}}$ was significantly higher than expected in all settings considered (Figure 4, Supplementary Table 2, see online supplementary material), with a relative excess of transmission from HCWs ranging from $31 \%$ to $76 \%$ more infections than expected. The proportion of transmission from HCWs was larger when considering infection of patients $(79.0 \%$; $95 \% \mathrm{Cl}$ 78.5-79.5\%), particularly frail patients (CFS score $>5: 82.3 \%$; 95\% Cl 81.8-83.4\%).

The sensitivity analyses did not show major changes in either the probabilities of transmission pairs or the overall structure of the consensus transmission tree, with the exception of the model without any contact data, which, as expected, had worse resolution and suggested fewer transmission events by HCWs (Supplementary Figures $4-7$, see online supplementary material). All sensitivity analyses similarly showed that the infections from HCWs were more frequent than usual (all $P$-values $<2.2 \mathrm{e}-16$ ), albeit with varying effect sizes (Supplementary Figure 8, see online supplementary material). Lowest effects were found when ignoring contact data, with an overall $f_{\mathrm{HCW}}$ of $54.9 \%(95 \% \mathrm{Cl} 54.5-55.3 \%)$, yet significantly greater than the expected $46.8 \%$ (Wilcoxon-Mann-Whitney test: $\mathrm{V}=480614, P<2.2 \mathrm{e}-16$ ).

\section{Case-control study}

Characteristics of patients with nosocomial COVID-19 and uninfected controls are shown in Table I. In the univariable analysis, patients with nosocomial COVID-19 were significantly older than uninfected controls [87 years (IQR 77-89) vs 80 (IQR $74-86)$ ], were less likely to be active smokers ( $2.7 \%$ vs $16.2 \%)$, and were more frail (CFS score $>5: 89.2 \%$ vs $54.1 \%$ ). There were no significant differences between nosocomial cases and uninfected controls in terms of obesity, delirium on admission, or prevalence of comorbidities, except for a lower proportion of cases with chronic respiratory disease among patients with nosocomial COVID-19 (16.2\% vs $33.8 \%$ ).

Results of the univariable and multi-variable logistic regression model for risk of acquisition of nosocomial COVID-19 are shown in Table III. In the multi-variable analysis, CFS score $>5$ substantially increased the risk of nosocomial COVID-19 (adjusted OR 6.94; 95\% Cl 2.13-22.57).

\section{Discussion}

This study describes an explosive nosocomial outbreak of SARS-CoV-2 in a rehabilitation clinic involving both patients and HCWs. Despite rich data and sequencing availability, including $\mathrm{HCWs}$, and even using advanced epidemiological and genomic analyses, the authors were unable to reconstruct who infected whom in the outbreak with high confidence. However, it was shown that HCWs played an undisputable role in introducing SARS-CoV-2 into the facility, and were the main drivers of infection to patients and each other.

It has been conventional wisdom, particularly during the first pandemic wave, that patients posed a greater risk to HCWs than vice versa, even with appropriate personal protective equipment. This has also been suggested by a recent outbreak investigation in an acute care hospital [28]. Although the authors do not dispute the fact that appropriate IPC measures are essential to protect HCWs, these results suggest that patients are more likely to become infected by HCWs than vice versa in non-COVID settings. HCWs did not only introduce SARSCoV-2 in the clinic, but were also at the origin of most superspreading events. Conversely, direct patient-to-patient transmission in a setting such as a rehabilitation clinic or LTCF does not appear to be a major driver of infection.

These results suggest that in such closed settings, with a dense outbreak and a relatively slowly evolving pathogen, genomic sequencing data offer little added value. Indeed, in this outbreak, the large majority of isolates formed a single large cluster. Nevertheless, genomic surveillance can still be useful, for example for the detection of novel variants of disease, and to understand epidemic and evolutionary patterns at larger geographical and temporal scales $[29,30]$.

Although this outbreak occurred in a setting with high adherence to traditional IPC measures, the authors managed to control the outbreak successfully with non-pharmaceutical interventions without availability of real-time genomic data. Indeed, $R_{t}$ decreased to $<1$ after closing the ward with the highest number of infections. The authors are confident that the IPC measures were responsible for the control of the outbreak, and not depletion of susceptibles. Indeed, given the peak estimated $R_{t}$ values, an attack rate ranging between $83 \%$ and $96 \%$ would have been expected in the absence of IPC measures, which is far greater than what was observed.

The findings from this study confirm previous concerns that $\mathrm{HCWs}$ play a major role in initiating, amplifying and sustaining outbreaks of nosocomial SARS-CoV-2 [6]. In this outbreak, approximately $80 \%$ of patient infection events were attributable to HCWs. Furthermore, HCWs can infect their peers in the work environment, but outside of direct clinical contact in places such as the cafeteria, break rooms or offices. Physical distancing guidelines can sometimes be difficult to adhere to due to architectural constraints (e.g. small offices). Practices such as car-sharing or couch-surfing due to border restrictions may also have played a role.

This study identified patient frailty as an important independent risk factor for nosocomial acquisition of SARS-CoV-2, which has been suggested by previous reports [31]. This can stem from biological phenomena whereby there is increased susceptibility to acquiring an infection (e.g. due to immunosenescence), or from differences in contact patterns because frail patients require more assistance for daily activities such 
A

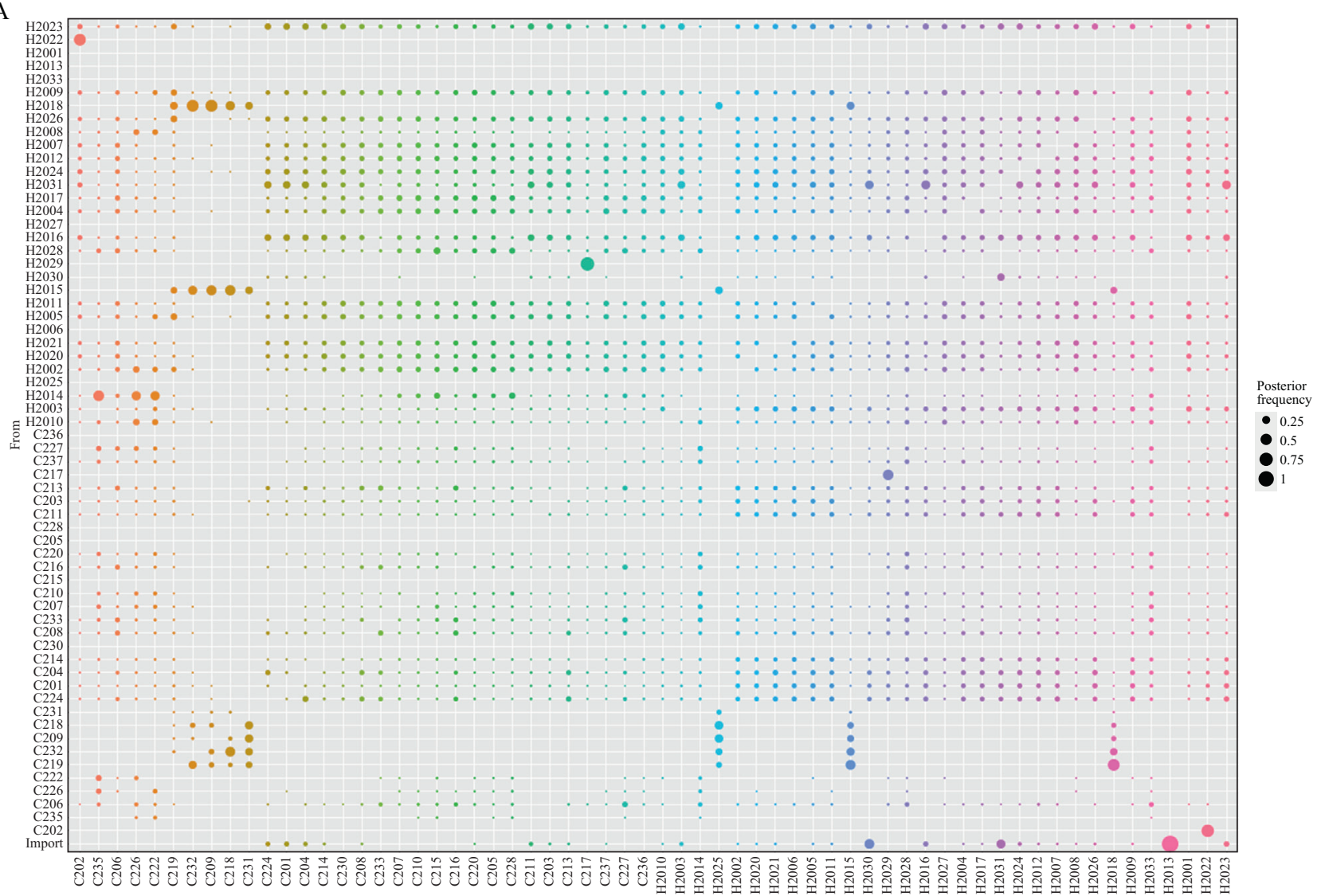

B

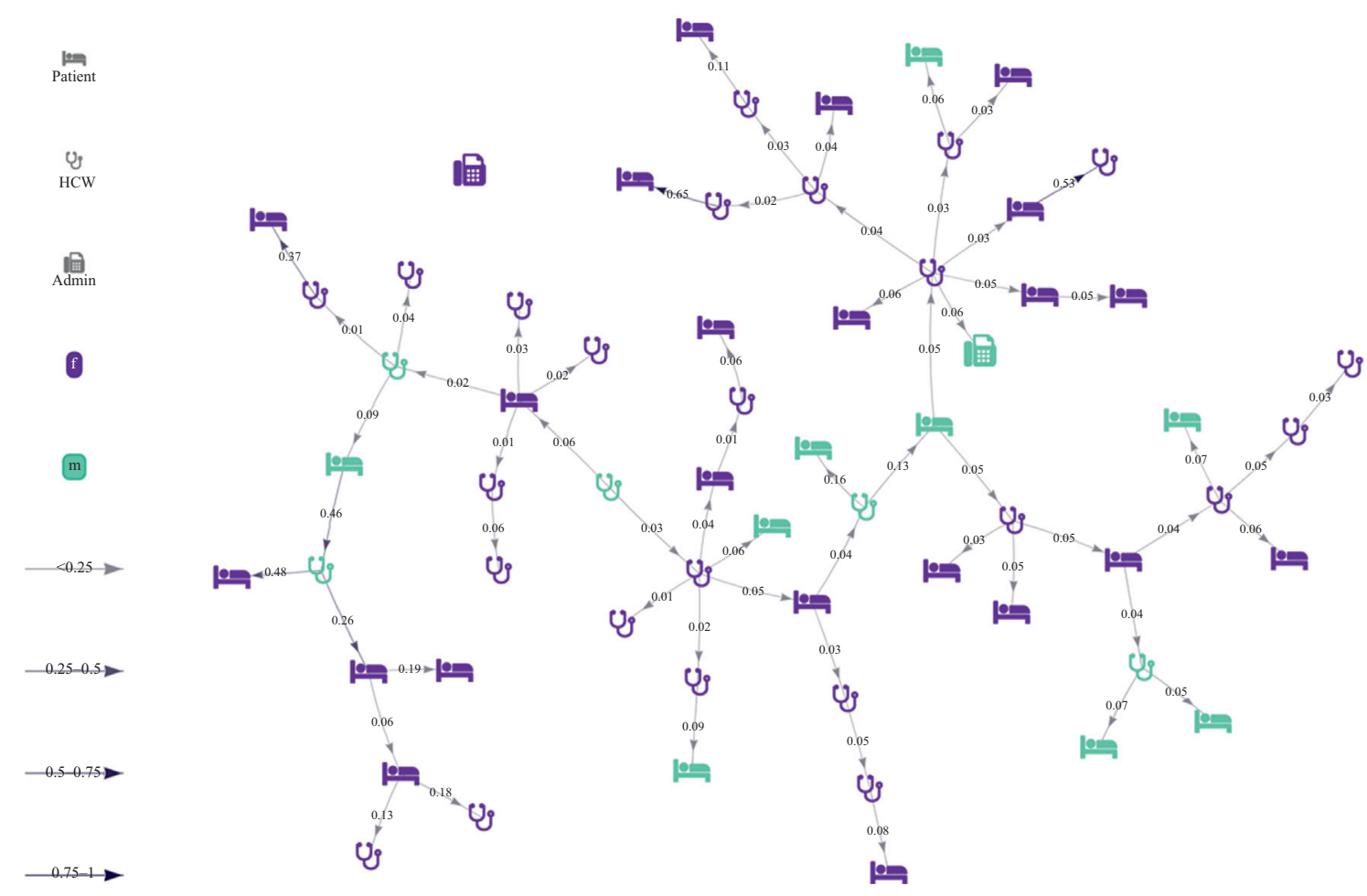

Figure 3. Selected output of the outbreaker2 model. (A) Ancestry reconstruction. (B) Transmission tree from Markov-Chain MonteCarlo iteration with highest likelihood. Patients and employees are named C2xx and H2Oxx, respectively. HCW, healthcare worker. 
as personal hygiene or dressing. Frailty in older patients with COVID-19, however, has been associated with poorer outcomes in geriatric patients [31,32], and this may be due to underlying biological reasons. For this reason, it is paramount that SARS-CoV-2 be kept outside the walls of LTCFs and nursing homes [33].

This study has several strengths, including the fact it was a comprehensive outbreak investigation which captured a high proportion of cases due to the aggressive testing strategy early in the first pandemic wave. Widespread testing of patients and HCWs, especially asymptomatic, is now conventional wisdom, but at the time represented a veritable paradigm shift in the management of respiratory viral nosocomial outbreaks during the early phase of the pandemic. Another strength of this study is that epidemiological and genomic data on HCWs who were part of the outbreak were included. Indeed, many outbreak investigations have few data or genomic sequences of HCWs [6], yet the present study demonstrated that their role is essential. The data collected in this study are of high quality as they were mostly collected prospectively. Also, the authors were able to obtain genomic sequences of SARS-CoV-2 for most $(>80 \%)$ cases, which increases the robustness of the approach. Finally, the authors used sophisticated modelling techniques, which combined epidemiological and genetic sequencing data in order to reconstruct the outbreak and to provide insight into transmission patterns.

Nevertheless, several limitations should be considered when interpreting the results. First, the estimates of serial interval and incubation periods that were selected can be challenged; however, the results were not sensitive to a longer serial interval, associated with less timely control of the outbreak. Second, not all cases were included in the outbreaker2

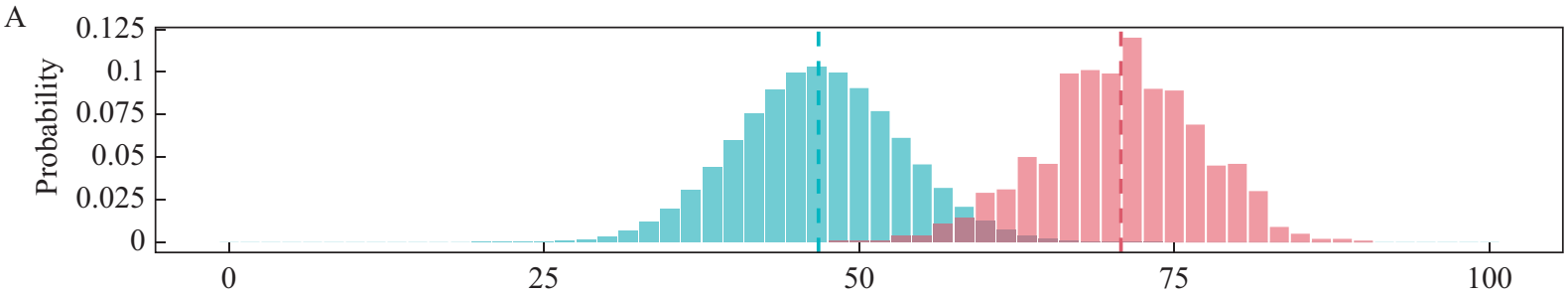

B

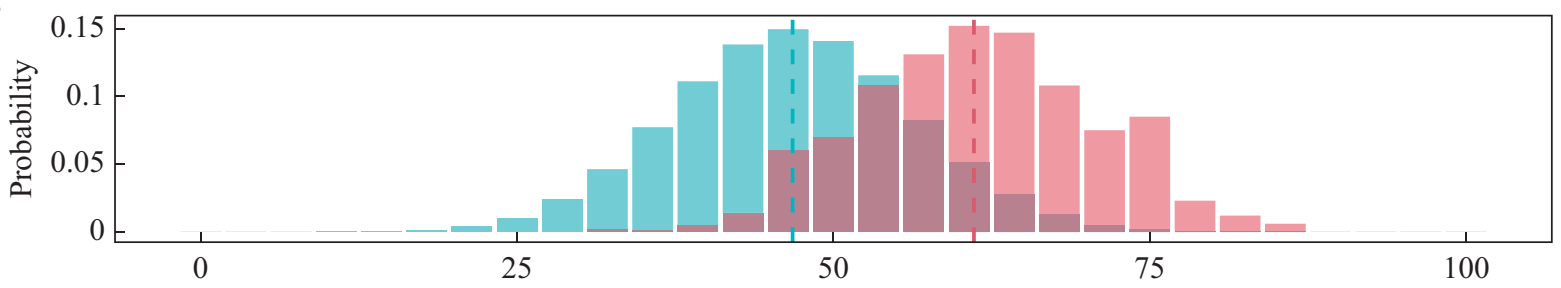

$\mathrm{C}$

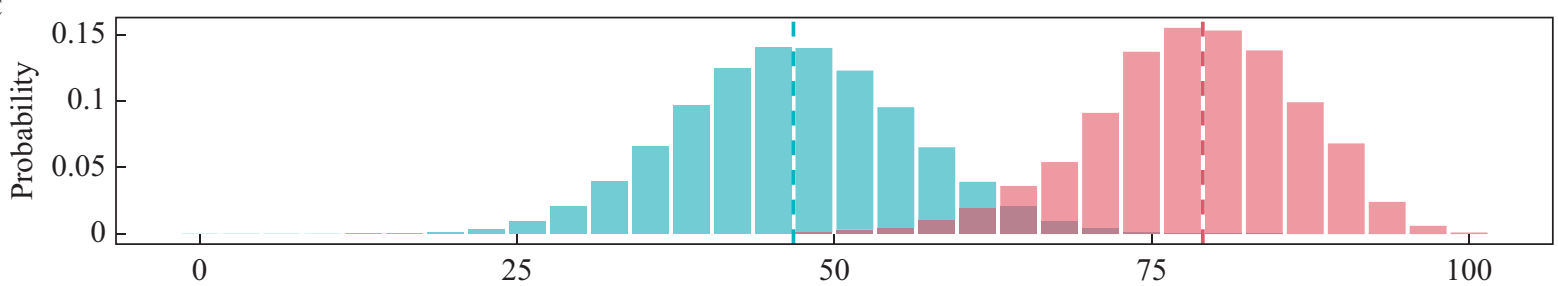

D

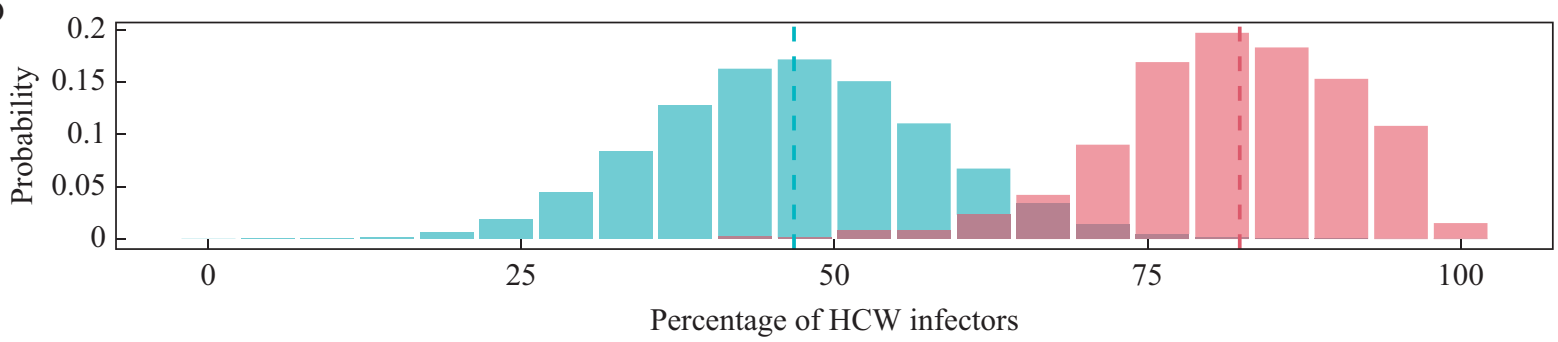

Figure 4. Proportions of transmissions attributed to healthcare workers $(\mathrm{HCWs})\left(f_{H C W}\right)$. The blue histograms indicate the expected binomial distributions of $f_{H C W}$ given the proportion of HCWs amongst cases. The red histograms show the distribution of $f_{H C W}$ across 999 transmission trees reconstructed by outbreaker2. Dotted lines indicate the mean estimate of the proportion. (A) All cases. (B) Transmission to HCWs alone. (C) Transmission to patients alone. (D) Transmission to frail patients alone. 
Table III

Univariable and multi-variable logistic regression analysis for risk of nosocomial acquisition of coronavirus disease 2019 among patients

\begin{tabular}{|c|c|c|c|c|}
\hline Characteristic & Unadjusted OR $(95 \% \mathrm{Cl})$ & $P$-value & Adjusted OR (95\% Cl) & $P$-value \\
\hline Age, years & $1.06(1.01-1.11)$ & 0.02 & & \\
\hline Age $(>80$ years $)$ & $1.73(0.77-3.88)$ & 0.18 & $1.03(0.42-2.52)$ & 0.95 \\
\hline Gender (female) & $1.55(0.65-3.69)$ & 0.32 & & \\
\hline Obesity & $0.48(0.15-1.56)$ & 0.22 & & \\
\hline Active smoking & $0.14(0.02-1.15)$ & 0.07 & & \\
\hline Clinical Frailty Scale score $>5$ & $7.01(2.26-21.79)$ & 0.001 & $6.94(2.13-22.57)$ & 0.001 \\
\hline Cumulative Illness Rating Scale - Geriatric $>14$ & $0.90(0.41-1.98)$ & 0.79 & & \\
\hline Charlson Comorbidity Index $>7$ & $2.46(1.07-5.65)$ & 0.03 & & \\
\hline Delirium on admission & $2.09(0.40-10.89)$ & 0.38 & & \\
\hline Diabetes mellitus & $0.49(0.18-1.34)$ & 0.16 & & \\
\hline Hypertension & $0.88(0.38-2.06)$ & 0.77 & & \\
\hline Chronic cardiovascular disease & $0.90(0.41-1.98)$ & 0.79 & & \\
\hline Chronic renal disease & $1.00(0.37-2.74)$ & 1.00 & & \\
\hline Chronic liver disease & $0.38(0.04-3.41)$ & 0.39 & & \\
\hline Chronic neurological impairment & $1.74(0.72-4.21)$ & 0.22 & & \\
\hline Cancer & $1.00(0.38-2.61)$ & 1.00 & & \\
\hline Dementia & $0.54(0.22-1.36)$ & 0.19 & & \\
\hline Immunosuppressive therapy & $1.00(0.09-11.40)$ & 1.00 & & \\
\hline Treatment with ACE inhibitor & $2.21(0.93-5.24)$ & 0.07 & & \\
\hline
\end{tabular}

$\mathrm{ACE}$, angiotensin-converting enzyme; OR, odds ratio; $\mathrm{Cl}$, confidence interval.

model due to lack of genomic sequences (14 of 76 cases); nonetheless, the model is designed and has been proven able to identify unsampled cases in the transmission chains [27].

In conclusion, this study showed that nosocomial outbreaks of SARS-CoV-2 in rehabilitation clinics can spread very quickly in a population with a naive immune system, and that both introduction and spread of disease can be mediated by HCWs. This has long-term implications for IPC in settings with older and frail populations who are at increased risk of acquiring respiratory viral infections, including SARS-CoV-2. This study also demonstrated an example of a situation where genomic data, even when augmented with rich epidemiological data, have little added value for outbreak control.

\section{Acknowledgements}

The authors wish to thank Rachel Goldstein (Geneva University Hospitals) for helping with data collection, Frédéric Bouillot (Geneva University Hospitals) for providing HR data, and Aurore Britan (Geneva University Hospitals) for data management. In addition, the authors would like to thank the team from the AMBUCoV study (Frédérique Jacquerioz Bausch, Julien Salamun, Hervé Spechbach). Romain Martischang (Geneva University Hospitals) provided the code for the figure of the interventions. Ashleigh Myall (Imperial College London) provided code to help generate the edge list of contacts. Finlay Campbell provided input on technical aspects of the outbreaker model development. Thibaut
Jombart receives funding from the Global Challenges Research Fund (GCRF) project 'RECAP' managed through RCUK and ESRC (ES/P010873/1), and from the MRC (Grant No. MC_PC_19065). Mohamed Abbas is supported by a grant from Geneva University Hospitals. Anne Cori is supported by the National Institute for Health Research (NIHR) Health Protection Research Unit in Modelling and Health Economics, a partnership between Public Health England, Imperial College London and LSHTM (grant code NIHR200908); and acknowledges funding from the MRC Centre for Global Infectious Disease Analysis (reference MR/R015600/1), jointly funded by the UK Medical Research Council (MRC) and the UK Foreign, Commonwealth \& Development Office (FCDO), under the MRC/FCDO Concordat agreement and is also part of the EDCTP2 programme supported by the European Union. Disclaimer: The views expressed are those of the author(s) and not necessarily those of the NIHR, Public Health England or the Department of Health and Social Care. The study team also wishes to thank the dedicated HCWs who looked after the patients.

\section{Conflict of interest statement}

None declared.

\section{Funding source}

This work was supported by a grant from the Swiss National Science Foundation under the NRP78 funding scheme (Grant No. 4078P0_198363). The funding source had no 
involvement in the writing of the manuscript or the decision to submit it for publication. All authors had full access to the full data in the study, and accept responsibility to submit for publication.

\section{Appendix A. Supplementary data}

Supplementary data to this article can be found online at https://doi.org/10.1016/j.jhin.2021.07.013.

\section{References}

[1] Griffin KM, Karas MG, Ivascu NS, Lief L. Hospital preparedness for COVID-19: a practical guide from a critical care perspective. Am J Respir Crit Care Med 2020;201:1337-44.

[2] Dora AV, Winnett A, Jatt LP, Davar K, Watanabe M, Sohn L, et al. Universal and serial laboratory testing for SARS-CoV-2 at a longterm care skilled nursing facility for veterans - Los Angeles, California, 2020. MMWR Morb Mortal Wkly Rep 2020;69:651-5.

[3] Arons MM, Hatfield KM, Reddy SC, Kimball A, James A, Jacobs JR, et al. Presymptomatic SARS-CoV-2 infections and transmission in a skilled nursing facility. N Engl J Med 2020;382:2081-90.

[4] Vanhems P, Saadatian-Elahi M, Chuzeville M, Marion E, Favrelle L, Hilliquin D, et al. Rapid nosocomial spread of SARS-CoV-2 in a French geriatric unit. Infect Control Hosp Epidemiol 2020;41:866-7.

[5] White EM, Santostefano CM, Feifer RA, Kosar CM, Blackman C, Gravenstein S, et al. Asymptomatic and presymptomatic severe acute respiratory syndrome coronavirus 2 infection rates in a multistate sample of skilled nursing facilities. JAMA Intern Med 2020;180:1709-11.

[6] Abbas M, Robalo Nunes T, Martischang R, Zingg W, Iten A, Pittet D, et al. Nosocomial transmission and outbreaks of coronavirus disease 2019: the need to protect both patients and healthcare workers. Antimicrob Resist Infect Control 2021;10:7.

[7] Taylor J, Carter RJ, Lehnertz N, Kazazian L, Sullivan M, Wang X, et al. Serial testing for SARS-CoV-2 and virus whole genome sequencing inform infection risk at two skilled nursing facilities with COVID-19 outbreaks - Minnesota, April-June 2020. MMWR Morb Mortal Wkly Rep 2020;69:1288-95.

[8] Sikkema RS, Pas SD, Nieuwenhuijse DF, O'Toole A, Verweij J, van der Linden A, et al. COVID-19 in health-care workers in three hospitals in the south of the Netherlands: a cross-sectional study. Lancet Infect Dis 2020;20:1273-80.

[9] Lucey M, Macori G, Mullane N, Sutton-Fitzpatrick U, Gonzalez G, Coughlan S, et al. Whole-genome sequencing to track SARS-CoV-2 transmission in nosocomial outbreaks. Clin Infect Dis 2021;72:e727-35.

[10] Lumley SF, Constantinides B, Sanderson N, Rodger G, Street TL, Swann J, et al. Epidemiological data and genome sequencing reveals that nosocomial transmission of SARS-CoV-2 is underestimated and mostly mediated by a small number of highly infectious individuals. J Infect 2021. https://doi.org/10.1016/ j.jinf.2021.07.034.

[11] Ellingford JM, George R, McDermott JH, Ahmad S, Edgerley JJ, Gokhale D, et al. Genomic and healthcare dynamics of nosocomial SARS-CoV-2 transmission. elife 2021. https://doi.org/ 10.7554/eLife.65453.

[12] Braun KM, Moreno GK, Buys A, Somsen ED, Bobholz M, Accola MA, et al. Viral sequencing to investigate sources of SARS-CoV-2 infection in US healthcare personnel. Clin Infect Dis 2021. https://doi.org/10.1093/cid/ciab281.

[13] Stone SP, Cooper BS, Kibbler CC, Cookson BD, Roberts JA, Medley GF, et al. The ORION statement: guidelines for transparent reporting of outbreak reports and intervention studies of nosocomial infection. Lancet Infect Dis 2007;7:282-8.
[14] Swissnoso Prevention and control of healthcare-associated COVID-19 outbreaks. Available at: https://www.swissnoso.ch/ fileadmin/swissnoso/Dokumente/5_Forschung_und_. Entwicklung/6_Aktuelle_Erreignisse/200515_Prevention_and_ control_of_healthcare-associated_COVID-19_outbreaks_V1.0_ ENG.pdf (last accessed September 2021).

[15] Thiabaud A, Iten A, Balmelli C, Senn L, Troillet N, Widmer A, et al. Cohort profile: SARS-CoV-2/COVID-19 hospitalised patients in Switzerland. Swiss Med Wkly 2021;151:w20475.

[16] Rockwood K, Song X, MacKnight C, Bergman H, Hogan DB, McDowell I, et al. A global clinical measure of fitness and frailty in elderly people. CMAJ 2005;173:489-95.

[17] Soeters PB, Wolfe RR, Shenkin A. Hypoalbuminemia: pathogenesis and clinical significance. J Parenter Enteral Nutr 2019;43:181-93.

[18] Charlson ME, Pompei P, Ales KL, MacKenzie CR. A new method of classifying prognostic comorbidity in longitudinal studies: development and validation. J Chronic Dis 1987;40:373-83.

[19] Salvi F, Miller MD, Grilli A, Giorgi R, Towers AL, Morichi V, et al. A manual of guidelines to score the modified cumulative illness rating scale and its validation in acute hospitalized elderly patients. J Am Geriatr Soc 2008;56:1926-31.

[20] Zekry D, Loures Valle BH, Graf C, Michel JP, Gold G, Krause KH, et al. Prospective comparison of 6 comorbidity indices as predictors of 1-year post-hospital discharge institutionalization, readmission, and mortality in elderly individuals. J Am Med Dir Assoc 2012;13:272-8.

[21] Corman VM, Landt O, Kaiser M, Molenkamp R, Meijer A, Chu DKW, et al. Detection of 2019 novel coronavirus (2019-nCoV) by realtime RT-PCR. Eurosurveillance 2020;25:2000045.

[22] Kumar S, Stecher G, Li M, Knyaz C, Tamura K. MEGA X: molecular evolutionary genetics analysis across computing platforms. Mol Biol Evol 2018;35:1547-9.

[23] Tamura K. Estimation of the number of nucleotide substitutions when there are strong transition-transversion and $\mathrm{G}+\mathrm{C}$-content biases. Mol Biol Evol 1992;9:678-87.

[24] Akaike H. A new look at the statistical model identification. IEEE Trans Automat Contr 1974;19:716-23.

[25] Cori A, Ferguson NM, Fraser C, Cauchemez S. A new framework and software to estimate time-varying reproduction numbers during epidemics. Am J Epidemiol 2013;178:1505-12.

[26] Jombart T, Cori A, Didelot X, Cauchemez S, Fraser C, Ferguson N. Bayesian reconstruction of disease outbreaks by combining epidemiologic and genomic data. PLoS Comput Biol 2014;10:e1003457.

[27] Campbell F, Didelot X, Fitzjohn R, Ferguson N, Cori A, Jombart T. outbreaker2: a modular platform for outbreak reconstruction. BMC Bioinform 2018;19(Suppl. 11):363.

[28] Klompas M, Baker MA, Rhee C, Tucker R, Fiumara K, Griesbach D, et al. A SARS-CoV-2 cluster in an acute care hospital. Ann Intern Med 2021;174:794-802.

[29] Volz E, Hill V, McCrone JT, Price A, Jorgensen D, O'Toole A, et al. Evaluating the effects of SARS-CoV-2 spike mutation D614G on transmissibility and pathogenicity. Cell 2021;184: 64-75.e11.

[30] Genomic sequencing in pandemics. Lancet 2021;397:445.

[31] Brill SE, Jarvis HC, Ozcan E, Burns TLP, Warraich RA, Amani LJ, et al. COVID-19: a retrospective cohort study with focus on the over-80s and hospital-onset disease. BMC Med 2020;18:194.

[32] Mendes A, Serratrice C, Herrmann FR, Genton L, Perivier S, Scheffler $M$, et al. Predictors of in-hospital mortality in older patients with COVID-19: the COVIDAge study. J Am Med Dir Assoc 2020;21:1546-54.e3.

[33] Robalo Nunes $T$, Lebowitz D, Fraccaro $M$, Perez M, Vieux L, Abbas $M$, et al. Can long-term care facilities remain a coronavirus disease 2019 (COVID-19)-free bubble? An outbreak report. Infect Control Hosp Epidemiol 2021;11:1-2. 